EchoGéo

\title{
Une lecture de politiques urbaines genrées dans des pays émergents
}

\section{Claire Hancock}

\section{(2) OpenEdition}

1 Journals

Édition électronique

URL : https://journals.openedition.org/echogeo/13145

DOI : $10.4000 /$ echogeo. 13145

ISSN : 1963-1197

\section{Éditeur}

Pôle de recherche pour l'organisation et la diffusion de l'information géographique (CNRS UMR 8586)

Référence électronique

Claire Hancock, "Une lecture de politiques urbaines genrées dans des pays émergents », EchoGéo [En ligne], 21 | 2012, mis en ligne le 10 octobre 2012, consulté le 11 août 2021. URL : http:// journals.openedition.org/echogeo/13145; DOI : https://doi.org/10.4000/echogeo.13145

Ce document a été généré automatiquement le 11 août 2021.

EchoGéo est mis à disposition selon les termes de la licence Creative Commons Attribution - Pas d'Utilisation Commerciale - Pas de Modification 4.0 International (CC BY-NC-ND) 


\title{
Une lecture de politiques urbaines genrées dans des pays émergents
}

\author{
Claire Hancock
}

1 Si l'on en croit les indicateurs des Nations Unies, la Corée du Sud a dépassé la France en 2006 en termes de développement humain. Aujourd'hui au $15^{\mathrm{e}}$ rang mondial derrière l'Islande, ce pays pourrait sembler hors du champ de ce qu'on appelle les "pays émergents". Pourtant, parmi les pays à développement humain très élevé, la Corée fait partie de ceux où les écarts d'éducation entre filles et garçons sont les plus élevés; il est aussi parmi ceux où l'activité salariée des femmes est la plus faible. Enfin, la représentation des femmes au parlement national n'arrivait en 2010 qu'à environ $17 \%$ de celle des hommes (soit moins qu'au Bahrein mais, il est vrai, plus qu'au Japon). Autant d'éléments qui doivent attirer l'attention sur le fait que le développement économique et humain peut s'accompagner de rigidités sociales, concernant notamment la participation des femmes aux activités économiques et la gestion politique, et partant, les rôles sociaux qui leur sont dévolus. Il ne s'agit pas d'argumenter ici sur un lien spécifique entre "l'émergence" de certains pays et la place des femmes dans ces pays, pas plus qu'on ne peut avancer d'explication convaincante en termes d'"aires culturelles": tout au plus l'exemple du Japon doit-il nous faire réfléchir sur l'hypothèse qui serait exagérément simpliste d'une résorption graduelle des inégalités genrées qui s'accomplirait en parallèle à la croissance économique ${ }^{1}$.

2 Je vais tâcher de montrer, à travers l'examen de deux cas dans deux villes de pays "émergents" (ou "issu de l'émergence", pourrait-on dire dans un cas...), Mexico et Séoul, comment ces questions peuvent se jouer dans de grandes agglomérations et au travers de certaines politiques spécifiques. Dans le premier cas, je m'appuie sur un travail de terrain, dans le deuxième, je ne dispose que de sources de seconde main, traduites de leur langue originale. Il me semble pourtant utile de pratiquer ce grand écart, très précisément pour échapper aux modèles explicatifs simplistes par "la culture" ou par "le développement économique", parce que ces deux exemples pris ensemble dévoilent un problème plutôt d'ordre politique, qui a à voir avec la prise en 
compte de la différence, et la reconnaissance de l'égalité dans la différence, et qui me semble avoir partie liée avec les mécanismes et contradictions de l'"émergence".

3 Je commencerai par un préalable assez substantiel sur les problèmes inhérents à la formulation de politiques urbaines ciblant le public féminin, et la posture intellectuelle qui peut permettre de s'extraire de la paralysie qu'induisent ces problèmes. J'aborderai ensuite l'exemple de Mexico, avant de mettre en évidence certaines spécificités du cas séoulite. On verra au travers de ces deux cas que de telles politiques posent plusieurs questions : est-ce à l'échelle des villes, si grandes et centralisées soient-elles, que doivent être traités des problèmes de parité ? Plus largement, est-ce en agissant sur l'espace qu'on peut résoudre des problèmes qui sont d'ordre social avant tout ? C'est au fond la question de la "mixité", souvent invoquée en France pour parler de mixité sociale, et posée comme antidote souverain à la ségrégation socio-spatiale, qui est posée, ici sous la forme de la mixité hommes/femmes.

\section{L'ambiguïté des politiques urbaines genrées}

4 Je m'intéresse dans cet article à ce que j'appelle des politiques urbaines "genrées", qui sont, en l'occurrence et comme c'est souvent le cas, des politiques à destination d'un public féminin. Je me trouve donc prise dans un mécanisme regrettable, qui a été bien décrit par Susan Fainstein et Lisa Servon (2005, p. 3) :

Gender is about roles and relationships, about differentials in power and access to resources. When we study gender, we are studying a system in which women, men, gays, lesbians and the trans-gendered are implicated and entwined. One cannot exist without the other. Gender has to do with the behaviors, expectations, and norms confronting each grouping in relation to the other. For this reason, it is critical that gender studies include men as subjects along with women. Still, most books and articles that use the word "gender" in their title focus almost exclusively on women. Why is this? One reason is clearly that women have been understudied in the past.

5 C'est également dans cet esprit que l'ouvrage récent de Guy Di Méo (2011) sur les pratiques urbaines féminines se donne pour but, dans un contexte français, de "rattraper ce temps perdu, cet oubli et cette injustice" (p. 5). Mais, à ne considérer que "les femmes" et leurs pratiques, on risque d'incriminer comme le fait Di Méo "l'espace social urbain (...) normatif (...) creuset actif d'attitudes sociales, d'idéologies, de formes originales de modes de vie, de pouvoirs"-en omettant de prendre en compte le "système" et les relations entre groupes et individus. Dans les politiques urbaines, on a souvent affaire à un discours et des représentations essentiellement masculins qui, lorsqu'ils n'occultent pas purement et simplement la différentiation genrée (l'usager, le passager, le riverain, sont toujours de pseudo-neutres pensés à l'image de décideurs en grande majorité masculins, très souvent aussi hétérosexuels, blancs, de classe moyenne et à mobilité "normale"), prétendent cibler un groupe "femmes" dont ils ont tout un ensemble d'idées normatives sur ce qu'"elles" font en ville ou ce qu'"elles" attendent de l'aménagement urbain.

6 Pour emprunter un exemple cité par Linda McDowell (2001), les aménageurs britanniques se sont flattés d'avoir répondu à une attente "des femmes" en installant des tables à langer dans les toilettes publiques pour femmes; il ne leur avait vraisemblablement pas traversé l'esprit que de jeunes pères puissent aussi avoir besoin de changer leurs enfants en bas âge dans l'espace public. On se trouve là en présence 
d'un cas type de politique éclairée et paternaliste tout à la fois : il s'agit en premier lieu de faciliter la fréquentation d'espaces publics par des mères de famille qui auraient pu auparavant les éviter à cause de la difficulté d'y prendre en charge certains besoins de leurs enfants. C'est en ce sens en première lecture une politique progressiste, qui prend en compte l'existence de publics différents, avec des besoins différents, et essaie de leur ménager un accès facilité. Mais en faire une politique "en faveur des femmes" simplement parce que la plupart du temps, les personnes accompagnant des enfants en bas âge dans l'espace urbain sont des femmes, c'est réitérer des schémas de genre figés et passéistes qui associent systématiquement public féminin et prise en charge des enfants. C'est à "la femme" dans son rôle de mère qu'on prête attention, sans se poser la question du danger de cette association systématique (qui redouble l'exclusion de celles qui ne le sont pas, autant qu'elle exclut pernicieusement les hommes de la prise en charge des enfants).

7 En ce sens, "prendre en compte les demandes des femmes" constitue une avancée sur la situation initiale où il paraît impensable aux aménagistes que différents publics puissent avoir différents besoins, et que l'accès à tous suppose de se débarrasser du modèle unique du citadin. De même, dans le milieu de la recherche, "faire entendre les voix des femmes" souvent étouffées, marginalisées, ou présentées comme déviantes par rapport à une norme implicite construite à partir du masculin, constitue vraisemblablement un progrès.

8 Mais le risque est également grand: on risque de réitérer, et de matérialiser dans l'espace urbain des schémas de différentiation entre hommes et femmes intrinsèquement problématiques, car liés à des rôles genrés eux-mêmes contestables et contestables par tous, j'insiste sur ce point, car la table à langer placée dans les toilettes des femmes est tout autant une atteinte au rôle dans l'éducation des enfants souhaité par certains pères, ou oncles, ou frères qu'elle est un cantonnement des femmes dans un rôle maternant.

Chercheurs et gestionnaires des villes se trouvent donc pris dans une contradiction, ou un paradoxe, somme toute similaire. Ce paradoxe a été souligné par de nombreuses auteures: c'est l'alternative entre "réforme" et "révolution" (ou pragmatisme et idéalisme). Soit on accepte que de petits aménagements dans les modes de vie sont souhaitables parce qu'ils facilitent, au quotidien, la vie des femmes, et que petit à petit, un meilleur accès à l'espace public, à l'emploi salarié, vont permettre un rapprochement entre leur statut social et celui des hommes (visibles en public et financièrement indépendantes, elles échappent à beaucoup des contraintes spatiales et contraintes familiales qui pèsent traditionnellement sur elles). Soit on refuse en bloc ces aménagements, précisément parce qu'ils apparaissent comme des dispensations d'en haut de pouvoirs paternalistes, et parce qu'ils rendent juste plus vivable au quotidien une inégalité de statut inacceptable, scandaleuse, qu'en somme ils contribuent à pérenniser un système injuste (qui cantonne les femmes dans des rôles de mère, de femme au foyer ou condamnée à une double journée de travail, etc.) - et que ce faisant ils retardent la nécessaire révolution contre ce système.

Ces deux positions sont suffisamment tranchées pour avoir conduit les féministes à se diviser profondément, entre tenantes de la "différence" (les femmes ont des besoins spécifiques, auxquels il convient d'appeler à répondre) et tenantes de l'"égalité" (les femmes sont en tous points les égales des hommes, et toute politique qui les cible attente à cette égalité). C'est ainsi que la loi sur la parité en politique, accueillie avec 
faveur par les premières qui y voyaient un moyen de remédier à la sous-représentation féminine, était dénoncée par les secondes affirmant que les femmes n'avaient pas besoin de passe-droit pour accéder aux fonctions électives.

11 Joan Scott a justement décrit la position des féministes françaises, depuis l'époque révolutionnaire, comme "paradoxale", et elle résume ainsi la situation :

Feminism was a protest against women's political exclusion; its goal was to eliminate "sexual difference" in politics, but it had to make its claims on behalf of "women" (who were discursively produced through "sexual difference"). To the extent that it acted for "women", feminism produced the "sexual difference" it sought to eliminate. This paradox-the need both to accept and to refuse "sexual difference"-was the constitutive condition of feminism as a political movement (1996, p. 3-4).

12 En s'inspirant de cette réflexion de Scott, Eric et Didier Fassin ont proposé d'étendre cette situation paradoxale à toutes les minorités, contraintes comme les femmes de "parler en tant que pour refuser d'être traité(e)s comme" (2006, p. 251) : en somme, il est nécessaire dans un premier temps d'affirmer la différence afin de voir reconnaitre son égalité. Les Fassin insistent à juste titre sur le fait que ce qui appelle reconnaissance n'est pas une quelconque spécificité culturelle: il n'existe pas plus de groupe "les femmes" intrinsèquement homogène et solidaire qu'il n'existe de groupe "les Noirs" homogène. Il s'agit alors d'en appeler à ce que Nancy Fraser appelle la "reconnaissance universaliste" (2000), c'est-à-dire la revendication d'une reconnaissance en tant que sujet politique égal à tous les autres, totalement distinct de la reconnaissance d'une spécificité ou d'une particularité culturelle ${ }^{2}$. Dans les termes de Mc Dowell (2001), il s'agit de ne pas succomber à un "essentialisme" qui postulerait une "essence" féminine réductrice et caricaturale, mais de mettre en œuvre un "essentialisme stratégique", qui tout en prenant en compte les différences entre les femmes, postule qu'on peut parler de solidarité entre elles et penser une "cause commune", avec pour objectif la lutte contre les discriminations subies par toutes à des degrés divers (et compliquées par ailleurs par d'autres discriminations fondées sur la classe, la race, l'orientation sexuelle, etc.).

\section{Retour à Mexico}

13 Ce détour par les réflexions nord-américaines, britanniques et françaises sur la dimension politique de l'affirmation de la "différence" nous permet de revenir mieux armés aux pays émergents et aux politiques mises en œuvre dans plusieurs grandes villes en direction du public féminin. Cet arsenal féministe et ces références puisées dans la philosophie politique et l'histoire sont en effet nécessaires pour savoir que penser de politiques sur lesquelles j'avoue que, pendant très longtemps, j'ai eu de la peine à me faire une opinion. Ils ont également pour vertu de rappeler que les sociétés de pays émergents peuvent être analysées avec des outils élaborés dans des contextes "émergés de longue date", ce qui n'est pas une opération d'impérialisme intellectuel occidental (Bourdieu et Wacquant, 1998), mais un refus de souscrire à des catégorisations lourdes de conséquences entre "Nord" et "Sud", Occident et nonOccident, etc. (Hancock, 2007).

14 J'effectue donc un retour sur le premier terrain que j'avais eu l'occasion d'effectuer en 1998 à Mexico, complété par des observations plus récentes effectuées pour les dernières au printemps 2011. J'ai rendu compte dans diverses publications de la 
situation qui avait en premier lieu, il y a treize ans, attiré mon attention : l'existence sur certaines lignes du métro de Mexico, aux heures de pointe, de voitures, de parties de quai et de couloirs de circulation réservés aux femmes (voir Hancock, 2000, 2002, 2004). Ce cas d'étude, dont j'ai eu l'occasion de rendre compte aussi devant différents publics, a toujours eu le don de susciter des réactions très vives et très partagées dans mon auditoire : le plus récent, celui du CIESAS de Mexico en avril 2011, s'étant avéré tout aussi divisé entre des habitant(e)s de la ville de Mexico et usagers des transports déclarant le système une "honte nationale" (car entérinant dans la gestion des transports ce qu'ils/elles dénonçaient comme le machisme ambiant et l'enfermement des deefeñas ${ }^{3}$ dans leur "différence" et leur vulnérabilité), et d'autres se félicitant du caractère pratique et confortable de la mesure-qui a été étendue à d'autres modes de transports, comme les lignes de bus en site propre (Metrobus), allant jusqu'à des "bus roses"4 et des "taxis roses" exclusivement féminins.

C'est évidemment une exception notable au constat fait par Fainstein et Servon, "(...) women had been invisible to transportation planners and (...) this invisibility produced an obliviousness to gendered differences in travel patterns" (2005, p. 8). C'est aussi, si on suit Jacqueline Coutras (1993), un enjeu important de l'affirmation des femmes que la conquête d'une mobilité en ville. C'est en tout cas, à Mexico, une politique qui a été engagée très précocement, puisque mon enquête initiale m'avait permis de retracer la mesure dans le métro à 1978. Elle se caractérisait comme une mesure paternaliste, décidée en haut lieu, et pas du tout concertée ni avec les groupes féministes, ni avec les usagers, dont les réactions ont été dès l'origine très mitigées. En se plaçant à un autre niveau d'analyse, on ne pouvait que constater que la mise en place de la mesure correspondait à une période de développement très rapide de l'emploi salarié féminin, qu'elle était très vraisemblablement censée accompagner ou accélérer. Dans une agglomération où les femmes représentent une très large majorité ( 91 hommes pour 100 femmes en 2010, d'après les chiffres de l'INEGI), un écart demeure entre le taux d'activité masculin ( $71,33 \%$ en 2010 ) et celui des femmes (43,76\%), mais ce dernier poursuit sa progression, et les femmes représentent de ce fait plus de $40 \%$ de la population active de la capitale. Il est naturellement impossible d'établir un lien de causalité entre la politique "genrée" et le développement du salariat féminin, tout au plus peut-on constater que les deux semblent aller de pair.

Il y a naturellement tout un ensemble d'aspects déplaisants à la séparation entre hommes et femmes dans le métro de Mexico, notamment le fait que les voitures et circulations séparées sont destinées, officiellement, non seulement aux femmes mais aussi aux personnes "vulnérables" (jeunes enfants, personnes âgées) : la métaphore de la "vulnérabilité" ayant souvent été utilisée pour exclure les femmes des espaces publics ou de certaines activités, il est compréhensible qu'elle hérisse les sensibilités féministes. Néanmoins, le fait que les protestations les plus vigoureuses émanent souvent du public masculin attire l'attention sur une autre caractéristique de la mesure : celle-ci n'est contraignante que pour les hommes, tandis qu'elle demeure optionnelle pour les femmes. Celles-ci sont libres de choisir d'aller dans les voitures "féminines" ou de rejoindre les voitures mixtes; les hommes, eux, n'ont pas ce choix (et des agents peu amènes les rappellent à l'ordre s'ils tentent de passer outre). Les dénonciations stridentes de "l'indignité" faite aux femmes résonnent donc souvent comme des blessures d'amour-propre masculin (car si les femmes sont renvoyées à leur "vulnérabilité", les hommes sont pour leur part renvoyés à une image infamante de machos incapables de se trouver en compagnie de femmes sans abuser de la situation, 
et cantonnés spatialement de ce fait). Le non-dit le plus notable autour de cette mesure, c'est l'absence de toute référence au harcèlement sexuel, ou aux attouchements susceptibles de se produire dans des transports en commun saturés, de la part des autorités de gestion des transports (STC) de Mexico. La question est pourtant présente à l'esprit de tous ceux qui discutent cette mesure, soit pour valider, soit pour mettre en cause son utilité. Les critiques formulées par les personnes de la classe moyenne s'appuient également souvent sur l'idée que séparer hommes et femmes ne peut être qu'un pis-aller, et que c'est toute une éducation sur les rapports hommes-femmes qui doit en fait être entreprise (auprès des classes populaires implicitement incriminées de sexisme) - que d'une certaine façon, les pouvoirs publics baissent les bras et renoncent à cette éducation, en entérinant dans les faits l'impossibilité de la mixité des publics aux heures où les transports sont les plus bondés.

\section{Séoul : le bonheur urbain}

17 Le moins qu'on puisse dire, c'est que la ville de Séoul s'est fendue d'une campagne de communication énergique autour de ses politiques genrées, puisqu'il en a été question dans la presse internationale. Souci d'éducation du public, ou souci de ne pas prendre par surprise comme visiblement le STP de Mexico s'était peu inquiété de le faire à la fin des années 70 ? En tout cas une bonne part du budget de plus de 100 millions de dollars mis en place en 2007 sous l'impulsion du maire Oh Se Hoon pour l'opération "Happy Women, Happy Seoul" semble avoir été consacrée à la communication autour du projet. Il est possible que ce soit le signe d'une plus grande maturité démocratique de la Corée du Sud de ce début de $21^{\mathrm{e}}$ siècle, ou du fait que le pays, interpellé de façon répétée par les organisations internationales et les économistes sur ses piètres résultats en termes de participation économique des femmes ${ }^{5}$, ait souhaité donner des gages à la communauté internationale. D'après les chiffres de l'OCDE, les femmes représentaient en $200941,6 \%$ de la population active en Corée, contre $37,5 \%$ au Mexique $^{6}$, par exemple. Une des singularités des femmes coréennes, qui les distingue très nettement des Mexicaines, c'est leur très faible indice de fécondité (1,2 enfants par femme soit l'un des plus faibles des pays de l'OCDE).

La politique "HWHS" a donc pris comme cible, d'après ses présentations officielles, deux publics féminins en particulier : les mères d'enfants en bas âge et les chômeuses. Ceci reflète sans doute un double objectif : inciter à un relèvement de la natalité et faire accéder plus de femmes à l'emploi salarié. Néanmoins, si l'on se réfère à un article de Veronica Zaragovia dans Time Magazine ("Will High-heel Friendly Streets Keep Seoul's Women Happy?", 5 août 2009), les mesures concrètes sont des plus diverses, et pas nécessairement en lien avec ces objectifs :

Assistant mayor of women and family policy affairs Cho Eun Hee says the program will be, among other things, helping to find work for jobless women, paving streets to make them high-heel friendly, building more women's public restrooms, improving lighting in public spaces, creating safe parks for women, expanding a women's taxi service and adding more public day-care centers. Cho says the project aims to eliminate "the inconveniences, anxiety and discomfort that women in Seoul experience on a daily basis."

Cet inventaire à la Prévert mérite qu'on s'y attarde: on y trouve en effet des dispositions contre lesquelles seules les plus acharnées des "égalitaristes" pourraient s'élever (aider les chômeuses à trouver un emploi, créer des places en crèche qui 
facilitent la conciliation de la maternité et de l'emploi salarié...), d'autres qui font bondir par leur stéréotypification des femmes (nécessairement porteuses de chaussures à hauts talons qui leur rendent la pratique des rues pavées peu aisée...), et d'autres enfin qui renvoient au sentiment d'insécurité que les femmes sont susceptibles de ressentir dans les lieux publics (aspect qui ne renvoie spécifiquement ni aux demandeuses d'emploi ni aux jeunes mères, mais bien aux risques de violences encourus par les femmes). Une autre mesure citée dans l'article consiste à peindre en rose des places de parking afin de réserver au femmes des stationnements plus proches des centres commerciaux ou centres d'affaires afin de minimiser leurs déplacements sans doute rendus pénibles par le port des fameux hauts talons qui inhibent notoirement la mobilité. Comme l'écrit assez sèchement la journaliste, "the plan may end up reasserting South Korean women's secondary status more than boosting it", et il est vrai qu'il en ressort une image de femmes victimes et proies sexuelles, fermement cantonnées dans leur fragilité. Or comme l'écrit justement M. Lieber "le sentiment d'insécurité déclaré par les femmes, qui dans les statistiques est généralement plus élevé que celui des hommes, n'est en aucun cas le simple prolongement logique d'une vulnérabilité naturelle ou essentielle des femmes. Au contraire, il fait partie intégrante de la construction socio-sexuée des identités" (2011), et il semble bien que des politiques ainsi présentées risquent de conforter cette construction.

Elles présentent également le risque de situer dans l'espace public urbain les principales restrictions à la mobilité et à la pleine participation économique et sociale des femmes, alors que c'est dans une large mesure le rôle conventionnellement attribué à ces femmes dans la société coréenne, de prise en charge des enfants et de "personne de la maison"7 - en d'autres termes, le front principal est celui de l'égalité dans l'espace domestique, et dans la famille, avant d'être une question d'aménagement urbain. Face à la crise économique de 2008, le premier réflexe du gouvernement coréen a été de favoriser l'emploi masculin au détriment des emplois féminins, conduisant à un déclin de la participation économique des femmes (Ahn, 2011).

21 Tout récemment (été 2011), le métro de Séoul s'est lancé dans une de ses énièmes tentatives pour créer des voitures réservées aux femmes (les précédentes, en 1992 et 1997, se seraient soldées par des échecs, du fait notamment du non-respect des consignes par le public, Eun, 2011). Contrairement à ce qui avait été le cas à Mexico, la motivation explicite de cette décision est la violence sexuelle, dûment recensée et mesurée comme telle (contrairement au métro de Mexico à la fin des années 70 qui ne recensait que "les agressions", et non leur caractère sexuel, pas plus que le genre de la personne qui en était victime). Mais comme à Mexico, la polémique fait rage, amplifiée par les réseaux sociaux en ligne assidûment fréquentés par les jeunes Séoulites (voir l'article de Eun sur Global Voices online, traduit en français sur le site Rue89).

\section{Conclusion : spatialiser pour désocialiser?}

Qu'une même mesure apparaisse nécessaire aussi bien dans la Séoul de 2011 que dans le Mexico de la fin des années 1970 doit en tout cas faire réfléchir8. A l'exception notable du Japon, tous les pays recensés comme pratiquant ce type de mesure par le site wikipédia appartiennent à la catégorie des pays émergents ou récemment émergés : Egypte, Inde, Iran, Taïwan, Brésil, Mexique, Indonésie, Dubaï et Philippines ${ }^{9}$. Ce sont en général des pays dont la croissance économique, accompagnée d'un ralentissement 
démographique plus ou moins marqué, a permis l'affirmation de classes moyennes aspirant à la démocratisation de leur société, mais rencontrant pour l'instant un inégal succès dans cette quête. La religion majoritaire dans ces pays semble peser relativement moins que leur tradition patriarcale fortement ancrée, comme le montre la juxtaposition dans cette liste de pays à majorité catholique, hindouiste, musulmane, bouddhiste, shintoïste...

On est en tout cas en présence de pouvoirs publics qui se posent le problème d'assurer la présence en public des femmes dans des conditions qui ne les exposent pas à des attitudes déplacées de la part du public masculin, préoccupation qui marque incontestablement un progrès par rapport à des situations où on considère en soi illégitime que des femmes se déplacent par elles-mêmes en ville. Il est vrai que l'émergence de ces pays se fait dans un contexte capitaliste où l'activité salariée des femmes est vue comme un moyen utile d'ajouter facilement des points à la croissance économique, alors même que celles-ci subissent des injonctions pour soutenir la croissance démographique et poursuivre leur travail de reproduction sociale. La question qui se pose est néanmoins celle soulevée aussi bien par des protagonistes mexicains que coréens : en traitant par des politiques spatialisées cette question des relations sociales de sexe, en créant ce que certains dénoncent comme "gender apartheid", n'est-on pas en train de retarder le recul du sexisme en dé-socialisant le problème? Une posture pragmatique conduit à considérer de telles mesures comme positives du fait des conséquences bénéfiques qu'elles sont susceptibles d'avoir sur le statut des femmes, là où une posture idéaliste amènerait à les condamner pour leurs limites évidentes.

Si l'on se réfère à l'idéologie de la "mixité sociale", la coexistence spatiale serait un gage de progrès démocratique et d'égalité de fait entre les membres d'une société : or, les études urbaines montrent bien que ce n'est pas si simple, et que se côtoyer dans les mêmes espaces ne résout en rien les inégalités, et peut même priver les plus modestes de certaines ressources de mobilisation politique (voir par exemple Tissot, Tévanian, 2004). Historiquement, l'entre-soi féminin a fait partie des revendications féministes, comme seul susceptible de garantir une prise de parole féminine autonome; il reste parfois revendiqué politiquement, dans bien des contextes où il n'est pas suspect d'être religieusement motivé (Tissot, Dupont, 2012). L'existence d'espaces ou lieux non-mixtes n'est donc pas dépourvue de pertinence, et l'on est peut-être en présence d'un des cas de figure où l'espace peut faire figure de ressource susceptible de transformer le social, même s'il importe de distinguer entre "non-mixité subie" (parfois au cœur même des structures qui se prétendent mixtes) et "non-mixité choisie", comme le fait Christine Delphy (Delphy, 2008).

\section{BIBLIOGRAPHIE}

Ahn S.-Y., 2011. Gender and space: a critical approach to women-friendly city projects in South Korea.Communication au 6ème colloque de géographie critique, Francfort, 18 août 2011. 
Bourdieu P., Wacquant L., 1998. Sur les ruses de la raison impérialiste. Actes de la Recherche en Sciences Sociales, vol. 121, $\mathrm{n}^{\circ}$ 1, p. 109-118.

Coutras J., 1993. La mobilité des femmes au quotidien. Un enjeu des rapports sociaux de sexe. Annales de la Recherche Urbaine, n59-60, p. 162-169.

Delphy C., 2008. La non-mixité, une nécessité. En ligne sur Les Mots sont importants, http:// lmsi.net/La-non-mixite-une-necessite

Di Méo G., 2011. Les Murs Invisibles. Femmes, genre et géographie sociale. Armand Colin, Recherches.

Eun L. Y., 2011. Polémique en Corée du Sud autour du métro pour femmes. Texte de Global Voices Online traduit par Jade Dussart, posté le 1er août sur www.rue89.com

Fainstein S. and Servon L. (eds), 2005. Gender and Planning. A Reader. New Brunswick, NJ, London, Rutgers University Press.

Fassin D., Fassin E. (dirs), 2006. De la question sociale à la question raciale. La Découverte.

Fraser N., 2000. Rethinking Recognition. New Left Review, n 3, mai-juin 2000, p. 107-120.

Hancock C., 2000. La séparation entre hommes et femmes dans le métro de Mexico : pour une régulation des flux? In M. Membrado et A. Rieu (dir), Sexes, espaces et corps. De la catégorisation du genre, Toulouse, éditions universitaires du Sud, p. 43-58.

Hancock C., 2002. Mujeres en la metropolí. Presencia femenina e imagen cinematográfica en la ciudad de México. Trace (revue du CEMCA, Mexico), $\mathrm{n}^{\circ} 42$ « Experiencia metropolitana y ciudadanía ", p. 65-70.

Hancock C., 2004. Le centre de Mexico, lieu d'émancipation des femmes mexicaines. In S. Denèfle (dir.), Femmes et villes, Presses de l’Université François Rabelais, p. 409-417

Hancock C., 2007. "Délivrez-nous de l'exotisme" : quelques réflexions sur des impensés de la recherche géographique sur les Suds (et les Nords). Autrepart, $n^{\circ} 41$, p. 69-81 in

McDowell L., 2001. Men, Women, Cities. In R. Paddison (ed), Handbook of Urban Studies, Sage.

Lieber M., 2011. Le sentiment d'insécurité au prisme du genre. Repenser la vulnérabilité des femmes dans les espaces publics. Métropolitiques, 5 décembre. URL : http://

www.metropolitiques.eu/Le-sentiment-d-insecurite-au.html

Scott J. W., 1996. Only Paradoxes to Offer. French Feminists and the Rights of Man. Cambridge, Mass., Harvard University Press.

Téllez Ballesteros, M. E., 2009. Planificacion y seguridad en el transporte urbano. Una vision de género en el caso de la ciudad de México. In R. Tello y H. Quiroz (eds.), Ciudad y diferencia. Género, cotidianedad y alternativas. Barcelona, ediciones Bellaterra, p. 13-126.

Tissot S., Tévanian P., 2004. La mixité contre le choix. En ligne sur Les Mots sont importants, http:// lmsi.net/La-mixite-contre-le-choix

Tissot S., Dupont N., 2012. Women only, men not allowed beyond this point. En ligne sur Les Mots sont importants, http://lmsi.net/Women-only-men-not-allowed-beyond 


\section{NOTES}

1. La dernière édition du Global Gender Gap Report du Forum Economique Mondial, qui classe les pays en fonction de l'importance des inégalités hommes-femmes, situe le Japon $98^{\mathrm{e}}$ sur 134 pays classés, la Corée du Sud $107^{\mathrm{e}}$ et le Mexique $89^{\mathrm{e}}$ (la France se classant $48^{\mathrm{e}}$ ).

2. On est ici tout proche de la pensée de Jacques Rancière, qui rappelle que "le seul universel politique est l'égalité" et qu'on peut sortir de l'opposition stérile entre identité et universalité en se souvenant que l'universalité n'est pas un abstrait, mais réside (et réside uniquement) dans le processus argumentatif par lequel l'égalité des uns (par exemple les femmes) avec les autres (par exemple les hommes) est établie. Voir Aux bords du politique, 1998, p. 116.

3. Habitantes du DF ou "District fédéral" qui accueille le centre de la ville de Mexico, mais désormais moins de la moitié de la population de l'agglomération dans son ensemble.

4. Programme "Atenea" mis en place en janvier 2008 (Téllez Ballesteros, 2009).

5. Par exemple, un article de The Economist, "What do you do when you reach the top?", soulignait que non seulement le taux d'activité féminin en Corée était un des plus faibles de l'OCDE, mais que ce taux était encore plus faible pour les femmes les plus diplômées, au contraire des autres pays, ce qui dénote un incontestable gaspillage de compétences qui pourraient être mises au service de la croissance économique (The Economist, 12 novembre 2011), dans un contexte où les femmes représentent la moitié des diplômés de niveau master (The Economist, 20 août 2011, "The flight from marriage").

6. L'écart avec les chiffres de l'INEGI cités plus haut s'explique par le fait qu'au Mexique, on mesure l'activité d'une classe d'âge plus étendue, dès 12 ans.

7. Expression utilisée par les hommes mariés pour se référer à leur femme, d'après une communication personnelle de V. Gelézeau que je remercie pour ses lumières sur la société coréenne en général...

8. Rappelons aussi que des voitures exclusivement féminines existaient par exemple également dans le métro de Londres, jusque dans les années 1960, et qu'il est périodiquement question de les instaurer à nouveau.

9. http://en.wikipedia.org/wiki/Women-only_passenger_car, page consultée le 27/12/2011.

\section{RÉSUMÉS}

Cet article propose une lecture critique de politiques urbaines "genrées" mises en place dans des grandes villes de pays émergents, en s'appuyant particulièrement sur les exemples de politiques visant le public féminin dans les transports en commun de Mexico et de Séoul. Après une première partie discutant de ce que peut être la posture féministe adéquate pour penser ces politiques, les deux cas d'étude sont passés en revue, afin d'en tirer des réflexions sur ce que de telles mesures nous disent des tensions sociales et politiques traversant les sociétés urbaines émergentes.

This paper critically assesses gendered urban policies implemented in large cities in emerging countries, with particular reference to policies facilitating the female public's access to public transportation in Mexico City and Seoul. After discussing the adequate feminist standpoint one can adopt, I review the case studies in order to illuminate some of the social and political tensions in emerging urban societies. 
INDEX

Mots-clés : genre, Mexico, politique urbaine, Séoul, transport

Keywords : gender, Mexico City, Seoul, transportation, urban policy

Thèmes : Sur le Champ - Sur le Terrain

\section{AUTEUR}

\section{CLAIRE HANCOCK}

Claire Hancock, hancock@mercator.ens.fr, est maître de conférences, Université Paris-Est Créteil (UPEC), Lab'Urba, IUF. Elle a publié récemment :

- Hancock C., Bret B., Gervais-Lambony Ph. et F. Landy (dir.), 2010. Justice spatiale et injustice spatiale. Presses de l'Université Paris-Ouest.

- Hancock C. (dir.), 2011. Genre, identités sexuelles et justice spatiale. Justice Spatiale/Spatial Justice, revue en ligne bilingue, $\mathrm{n}^{\circ} 3$, www.jssj.org/archives/03

- Hancock C., 2011. Le corps féminin, enjeu géopolitique dans la France postcoloniale. L'Espace Politique, $\mathrm{n}^{\circ} 14$, numéro coordonné par M. Blidon et $\mathrm{S}$. Roux, « Géo/politique du sexe », http:// espacepolitique.revues.org/index1882.html 